\title{
Ann-Trained Using Bat Algorithm for Modeling University-Based Energy Consumption on Short Term Basis
}

\author{
Okelola, Muniru Olajide ${ }^{1} \quad$ Amole Abraham Olatide ${ }^{2 *}$ \\ 1.Department of Electronic and Electrical Engineering, Ladoke Akintola University of Technology, P.M.B. \\ 4000, Ogbomoso, Nigeria \\ 2.Department of Electrical, Electronic and Computer Engineering, Bells University of Technology, P.M.B. 1015, \\ Ota, Ogun State, Nigeria
}

\begin{abstract}
Adequate planning and right decision making in the energy sector lies on accurate forecasts of the load demand. In this paper, Artificial Neural Network (ANN) trained via Bat algorithm was employed for short term load projection of University of Ibadan, Nigeria. Daily load demand of the study area was obtained from the log records. The neural network was built, trained with historical data gotten from the premier University in Nigeria and then used to predict 24 hour's load demand from Dec., $1^{\text {st }}$ to Dec., $7^{\text {th }}, 2016$. The experimental results indicated that the proposed method achieved a Mean Absolute Percentage Error (MAPE) of $6.60 \%$ and a Mean Percentage Error (MPE) of $4.17 \%$. This research finds application in scheduling of power demand in power system.
\end{abstract}

Keywords: Artificial Neural Network, Bat algorithm, Mean Absolute Percentage Error, Mean Percentage Error, Short-term forecasting,

DOI: $10.7176 /$ JIEA/10-2-04

Publication date:March $31^{\text {st }} 2020$

\section{Introduction}

In human race, electrical energy is a crucial and non-negotiable commodity known to have prop-up varieties of products and services meant to enhance the quality of life, promote productivity of workers as well as a positive catalyst encouraging wide range of entrepreneurial activities (Merkel, Povinelli, and Brow, 2018). Olabode et al., (2018) viewed electrical energy as a major energy resources required for efficient and effective running of day to day activities of household, commercial, and industrial sectors. However, energy-demand gap is a serious devastating challenge among the third world countries and one of the key indices by which energy managers / stakeholders can be kept abreast of impending surge in energy demand is load forecasting (Olabode et al., 2019). Load forecasting predicts intelligently what future load demand will be based on the historical load pattern of a particular location with acceptable reliability and satisfactory accuracy (Olabode et al., 2019). Not only does load forecasting enhances optimal scheduling of energy but also guaranteed appropriate operation of electric power system as whole (Jianwei, et al., 2018).

Projection of future load demand can be on short term, medium and long term. Each types of this load projection approach has its own areas of application in power system engineering, for instance short term load forecasting (STLF) is very efficient for scheduling of generation and transmission of energy (Olabode et al., 2019), Samuel et al., (2017) and Kalu et al., (2015) viewed STLF as the backbone of system operation and stability such as short-term unit maintenance work either for daily or weekly basis, minimization of cost associated with energy generation and efficient running of power system. STLF is a viable tool for assessing network reliability and as well as a means to lower incidences of failure in equipment and prevalence of blackouts (Olagoke, Ayeni, and Hambali, (2016). Of interest in this paper is short term load forecasting, several techniques have been reported in literatures for solving this type of load forecasting which include exponential smoothing, multiple regression, stochastic time series, exponential smoothing technique, iterative weighted least squares and artificial intelligence techniques (Olagoke, Ayeni, and Hambali, (2016).

STLF of Nigerian power system was addressed with the aid of artificial neural network (ANN) on daily basis using previous load consumption pattern for the month of August in the year 2003, ANN was trained via multilayer perception with the previous load data of the study location, the work established that insufficient hidden neutron makes it difficult to learn the series behaviour while too many hidden neutrons make the system to be over trained, the authors concluded that high degree of accuracy was achieved with the proposed approach on the average (Sheikh and Unde, 2012). Adepoju et al., (2007) employed ANN and genetic algorithm (GA) to forecast $330 / 132 / 33 \mathrm{kV}$ substation, Ganmo, Kwara state south-west Nigeria on short term using the load data of May, 2014; GA was engaged to train the hidden layers of ANN while Levenberg Marquardt was engaged to train the whole model of ANN, the work was able to establish accurately the non-linear relationship existing between the temperature and historical load of the study location.

Similarly, the load projection of Kenya national power grid was carried out using supervised ANN-based model with additive regression as classifier on short term (Christopher and Francis, 2013). Also, Uduak,et al., (2018) employed interval type 2 fuzzy logic-ANN to address STLF using the load data of Uyo, Akwa Ibom State, 
south-south Nigeria, ANN was trained with back propagation so as to reduce the error-based function. The authors submitted that interval type 2 fuzzy logic-ANN was able to handle the uncertainties and flexibly tuned the interval type 2 fuzzy logic parameters which resulted in better forecasting of the study case with minimized error in forecast. Having examined the earlier works reported with the use of ANN on Nigerian grid, this works therefore aim to extend the effort of earlier works by using Bat Algorithm to train the hidden layer of ANN. The rest of this paper is organized thus; material and methods were discussed in section 2, result and discussion was presented in section 3 while section 4 presented the conclusion.

\section{Material and Methods}

This section presents the material and methods adopted in the course of this work.

\subsection{Data Collection}

A non-weather data was used for this work, a daily energy consumption of the University of Ibadan as contained in the daily hourly load reading sheets for a week was collected as shown in Table 1.

\subsection{Artificial Neutral Network Structure}

ANN is a computational model that is structured to function in similitude of biological neural networks. ANN is endowed with capability to learn from training data set and this feature of ANN is termed adaptive learning. After learning by training which can be supervised or unsupervised, it creates its own structure by way of selforganization. Three stages are involved in ANN: training, testing and validation. In this present work, the whole data set was divided into two sets: training set and testing set. Training set consists of $80 \%$ of whole data and testing set contains the rest data. The training set was used to make a model which, therefore, predicts the load in the future. The number of hidden neuron was automated with the aid of Bat Algorithm (BA). The parameter sets of BA used in this work is as shown in Table 2 and the summary of ANN structure used in this work is as shown in Table 3.

Table 1: Daily Load Demand of University of Ibadan, Nigeria for Dec., $1^{\text {st }}-7^{\text {th }}, 2016$

\begin{tabular}{|c|c|c|c|c|c|c|c|}
\hline \multirow{3}{*}{$\begin{array}{l}\text { Time } \\
(\mathrm{hr})\end{array}$} & \multicolumn{7}{|c|}{ Daily Load Demand (MW) } \\
\hline & \multirow{2}{*}{\multicolumn{3}{|c|}{ 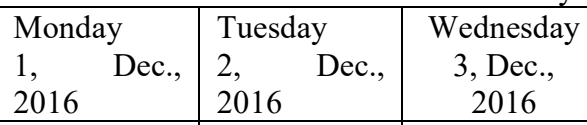 }} & \multirow{2}{*}{$\begin{array}{l}\text { Thursday } \\
4, \quad \text { Dec, } \\
2016\end{array}$} & \multirow{2}{*}{$\begin{array}{l}\text { Friday } \\
5, \quad \text { Dec, } \\
2016\end{array}$} & \multirow{2}{*}{$\begin{array}{l}\text { Saturday } \\
6, \quad \text { Dec, } \\
2016\end{array}$} & \multirow{2}{*}{$\begin{array}{l}\text { Sunday } \\
7, \quad \text { Dec, } \\
2016\end{array}$} \\
\hline & & & & & & & \\
\hline $1: 00$ & 1.65 & 1.91 & 0.00 & 1.73 & 1.73 & 1.53 & 1.55 \\
\hline $2: 00$ & 1.56 & 1.71 & 0.00 & 0.00 & 1.55 & 1.82 & 1.40 \\
\hline $3: 00$ & 1.47 & 1.70 & 0.00 & 0.11 & 1.55 & 1.65 & 1.37 \\
\hline $4: 00$ & 1.46 & 1.65 & 0.00 & 1.24 & 1.51 & 1.48 & 1.37 \\
\hline $5: 00$ & 1.56 & 1.68 & 0.00 & 1.68 & 1.50 & 1.52 & 1.49 \\
\hline $6: 00$ & 1.63 & 1.78 & 0.00 & 1.71 & 1.70 & 1.64 & 1.51 \\
\hline $7: 00$ & 1.01 & 1.97 & 0.00 & 1.74 & 1.77 & 0.18 & 1.59 \\
\hline $8: 00$ & 2.41 & 2.65 & 0.00 & 1.65 & 2.68 & 0.28 & 2.08 \\
\hline $9: 00$ & 4.29 & 4.37 & 0.00 & 1.64 & 3.86 & 1.71 & 3.72 \\
\hline $10: 00$ & 0.00 & 4.89 & 0.00 & 1.62 & 4.30 & 3.68 & 4.43 \\
\hline $11: 00$ & 1.74 & 5.07 & 0.00 & 0.16 & 4.38 & 0.43 & 4.31 \\
\hline $12: 00$ & 0.00 & 5.08 & 0.00 & 0.16 & 0.00 & 2.28 & 4.64 \\
\hline $13: 00$ & 0.00 & 4.97 & 0.00 & 0.32 & 0.00 & 0.00 & 4.71 \\
\hline $14: 00$ & 0.83 & 5.00 & 0.00 & 0.37 & 0.00 & 1.67 & 0.70 \\
\hline $15: 00$ & 0.76 & 0.72 & 0.00 & 0.41 & 0.00 & 1.66 & 0.48 \\
\hline $16: 00$ & 1.36 & 0.00 & 0.25 & 1.31 & 0.00 & 1.83 & 0.57 \\
\hline $17: 00$ & 1.71 & 0.00 & 2.10 & 0.00 & 0.00 & 0.46 & 0.30 \\
\hline $18: 00$ & 1.45 & 0.26 & 0.00 & 0.00 & 0.00 & 0.43 & 0.75 \\
\hline $19: 00$ & 1.86 & 0.00 & 1.59 & 0.14 & 0.00 & 0.46 & 1.42 \\
\hline $20: 00$ & 3.27 & 0.92 & 1.76 & 2.17 & 0.88 & 0.42 & 1.65 \\
\hline 21:00 & 0.24 & 2.48 & 2.43 & 2.14 & 2.49 & 1.58 & 2.16 \\
\hline $22: 00$ & 2.35 & 2.41 & 2.28 & 2.09 & 0.48 & 2.23 & 2.47 \\
\hline $23: 00$ & 2.14 & 2.19 & 2.07 & 2.25 & 0.00 & 2.00 & 2.25 \\
\hline $00: 00$ & 2.12 & 0.00 & 1.93 & 2.04 & 0.34 & 1.73 & 2.08 \\
\hline
\end{tabular}


Table 2: Bat Algorithm Parameters

\begin{tabular}{|c|l|l|}
\hline S/N & \multicolumn{1}{|c|}{ Bat Algorithm } & Optimal Values of BA Parameter \\
\hline 1 & Population Size & 20 \\
\hline 2 & Number of generations & 1000 \\
\hline 3 & Loudness & 0.5 \\
\hline 4 & Pulse rate & 0.5 \\
\hline 5 & Frequency minimum & 0 \\
\hline 6 & Frequency maximum & 2.0 \\
\hline 7 & Number of dimensions & 10 \\
\hline
\end{tabular}

Table 3: Summary of ANN Parameters

\begin{tabular}{|c|l|l|}
\hline S/N & \multicolumn{1}{|c|}{ ANN } & \\
\hline 1 & Network Topology & Multi-layer feed forward ANN \\
\hline 2 & Variable used as input & $\begin{array}{l}\text { hour of the day, day of the week, and daily } \\
\text { load demand }\end{array}$ \\
\hline 3 & No of hidden neutrons & Automated using bat algorithm \\
\hline 4 & No of iterations & 100 \\
\hline 5 & Training algorithm & Bat Algorithm \\
\hline 6 & Hidden layer transfer function & Sigmond \\
\hline 7 & Output layer transfer function & Sigmond \\
\hline
\end{tabular}

\subsection{Performance Metrics}

The prediction accuracy is a good approach to measure the accuracy of artificial neutral network. In this work, mean percentage error (MPE) and mean absolute percentage error (MAPE) was used to assess the accuracy of the forecast. The least value of MPE and MAPE is an indication of optimum performance of the proposed techniques. Mean Percentage Error;

$$
M P E=\frac{1}{N} \sum_{i=1}^{N} \frac{\left(x_{t}-x_{f}\right)}{x_{t}} \times 100 \%
$$

Mean Absolute Percentage Error

$$
M A P E=\frac{1}{N} \sum_{i=1}^{N} \frac{\left|x_{t}-x_{f}\right|}{x_{t}} \times 100 \%
$$

where, $x_{t}=$ real load at time $(t), x_{f}=$ forecasted load at $(t)$ time.

\section{Result and Discussion}

This section presented the outcome of this research which aimed to project the hourly load demand of a typical university environment. The study case used is the premier University; University of Ibadan, Ibadan, Oyo State Nigeria. Figure 1 to Figure 7 depicted the actual and forecasted load in Megawatts (MW) for each day in the month of December, 2016 were plotted against the time in hours.

Figures 1 to 7 show that ANN model developed produced an output that is much closer to the actual output. It was observed that the peak load values for Tuesday, Wednesday, Thursday and Friday were high due to the considerable operations of residential and commercial loads while the peak load value for Monday is lower due to its proximity to the weekends. Saturday and Sunday being the weekends have the lowest peak value due to negligible operation of commercial and industrial loads within the university environment. 


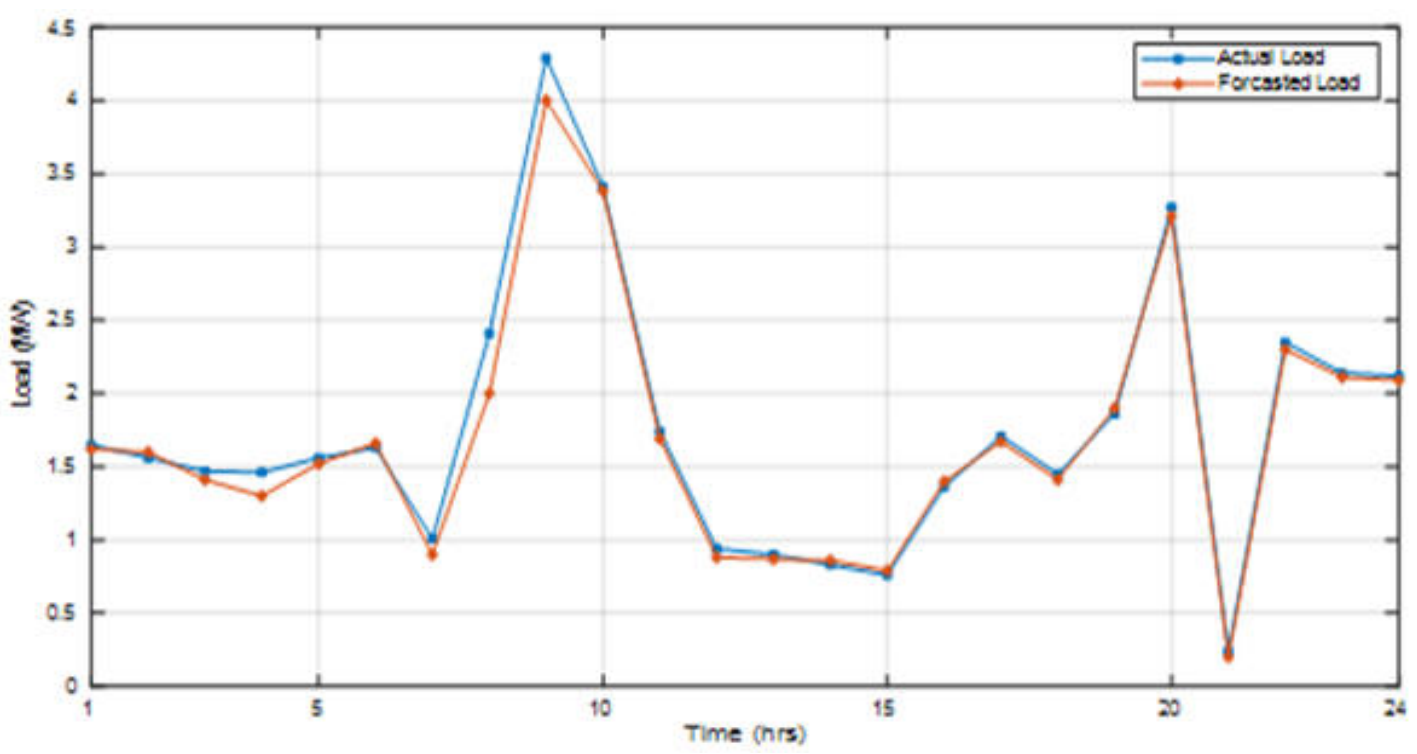

Figure 1: Monday, $1^{\text {st }}$ December 2016 hourly load curve

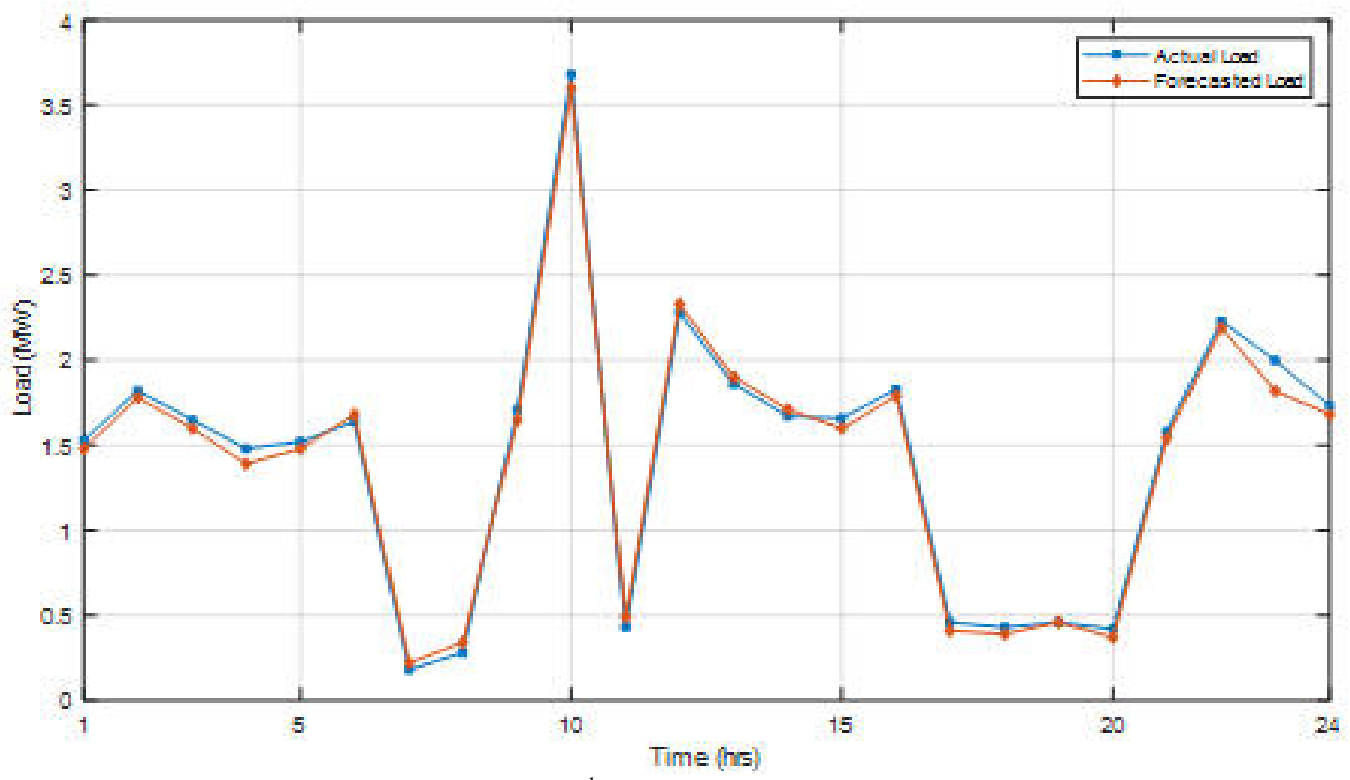

Figure 2: Tuesday, $2^{\text {nd }}$ December 2016 hourly load curve 


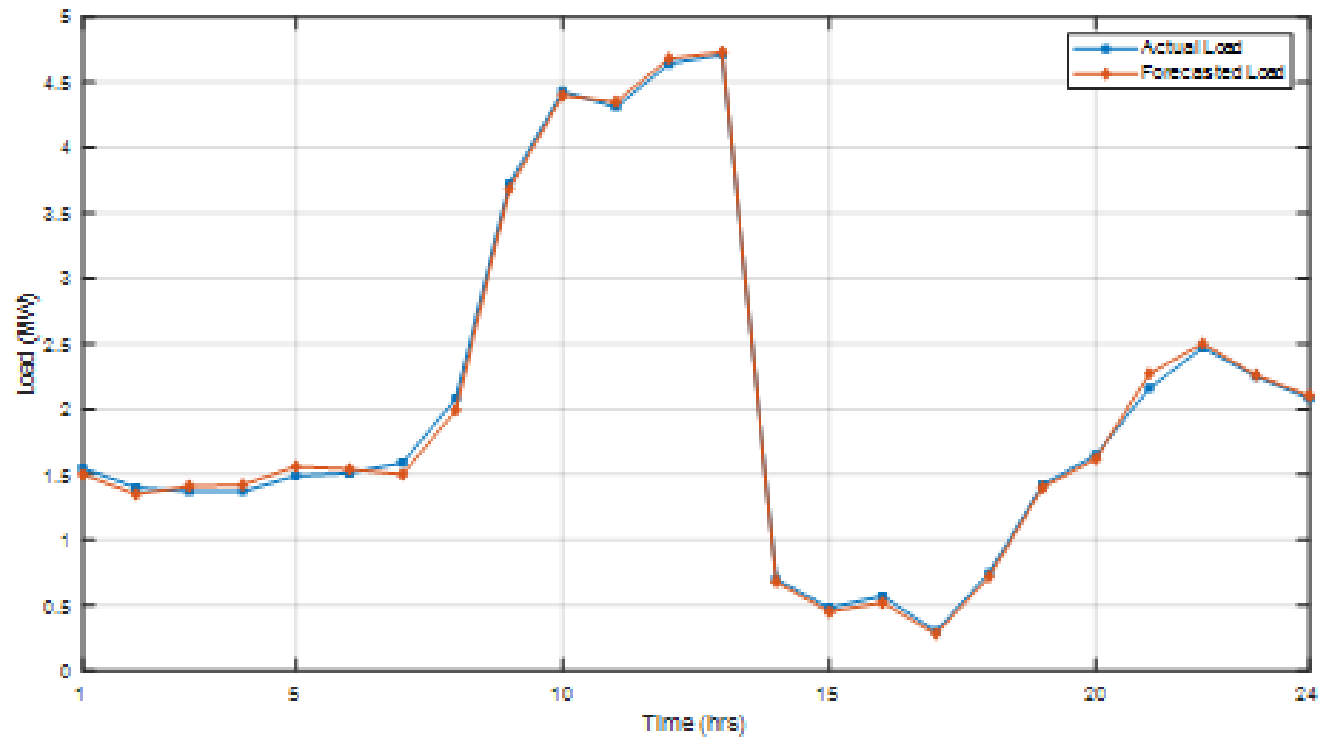

Figure 3: Wednesday, $3^{\text {nd }}$ December 2016 hourly load curve

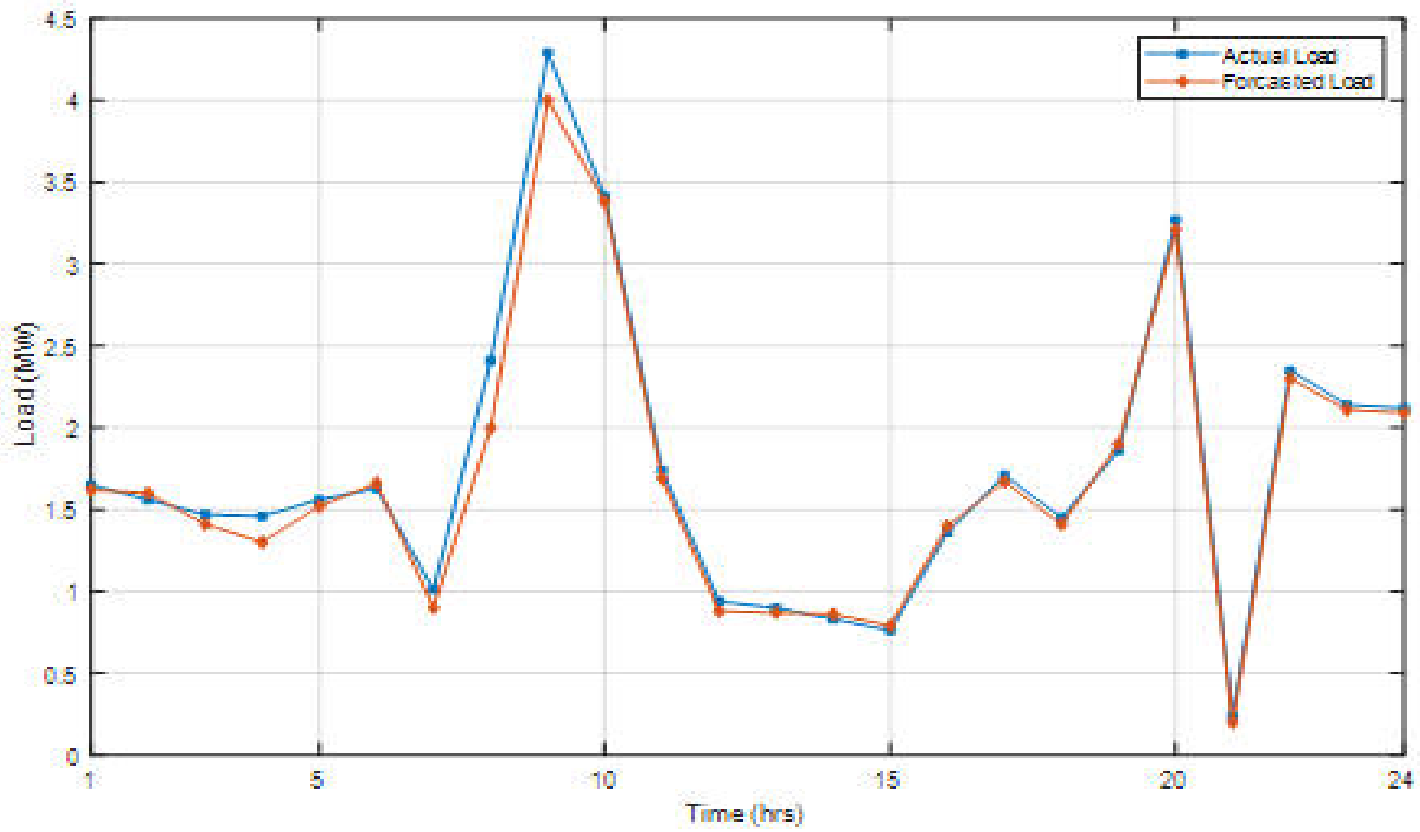

Figure 4: Thursday, $4^{\text {rd }}$ December 2016 hourly load curve 


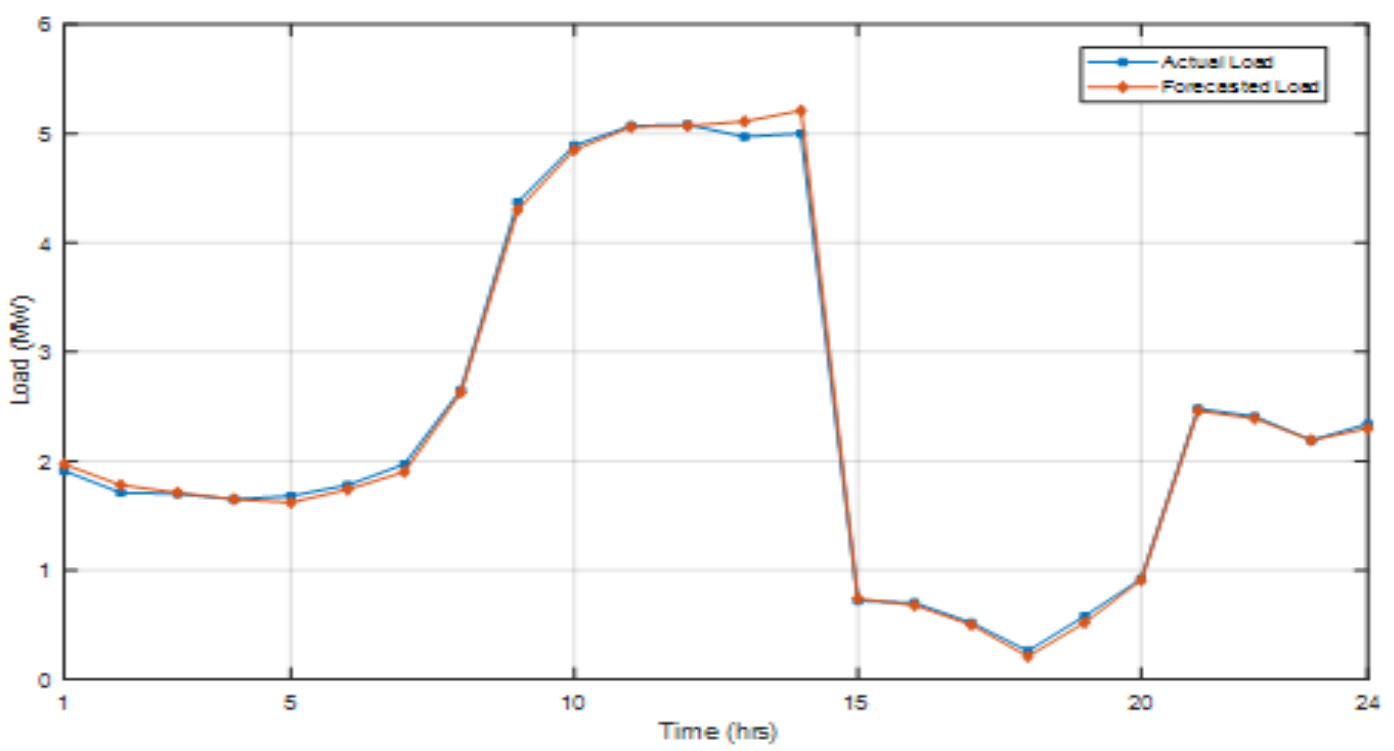

Figure 5: Friday, ${ }^{\text {th }}$ December 2016 hourly load curve

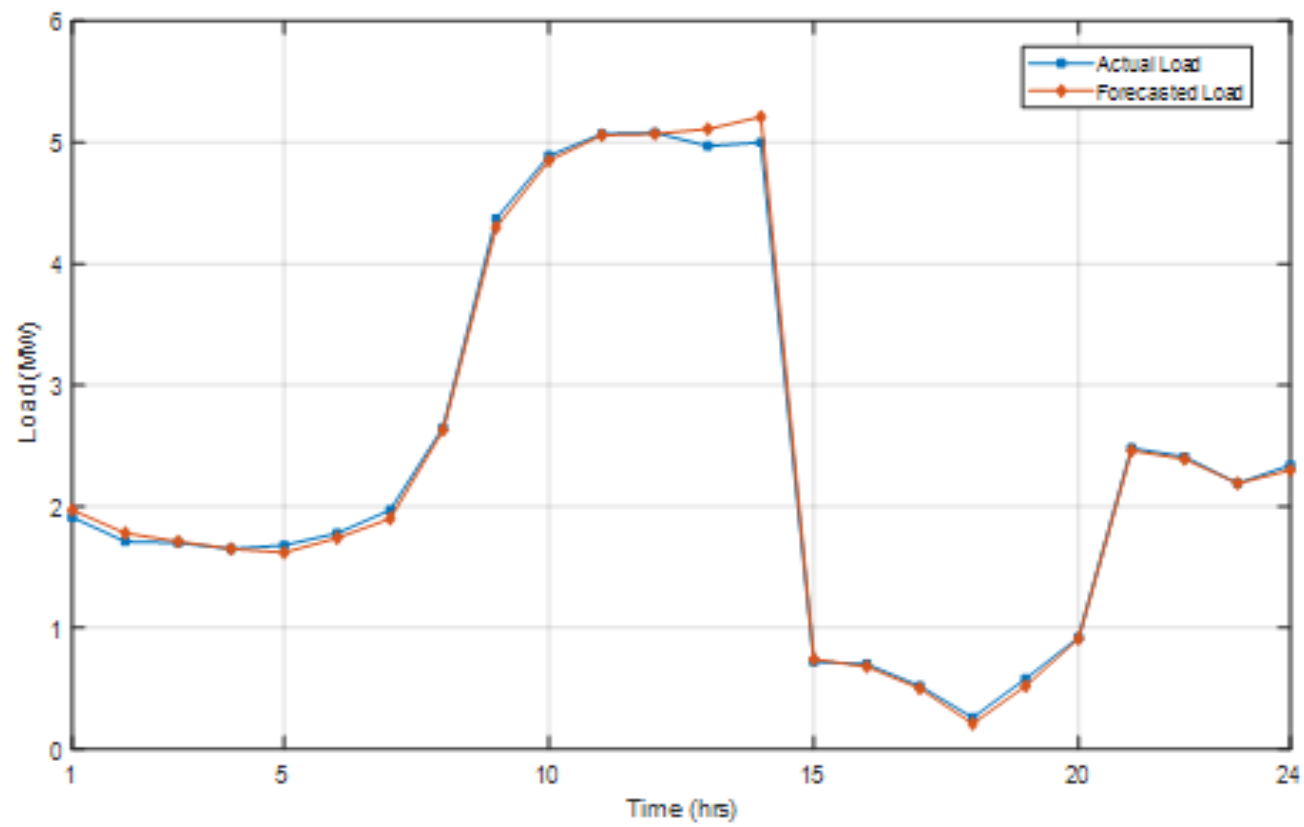

Figure 6: Saturday, $6^{\text {th }}$ December 2016 hourly load curve 


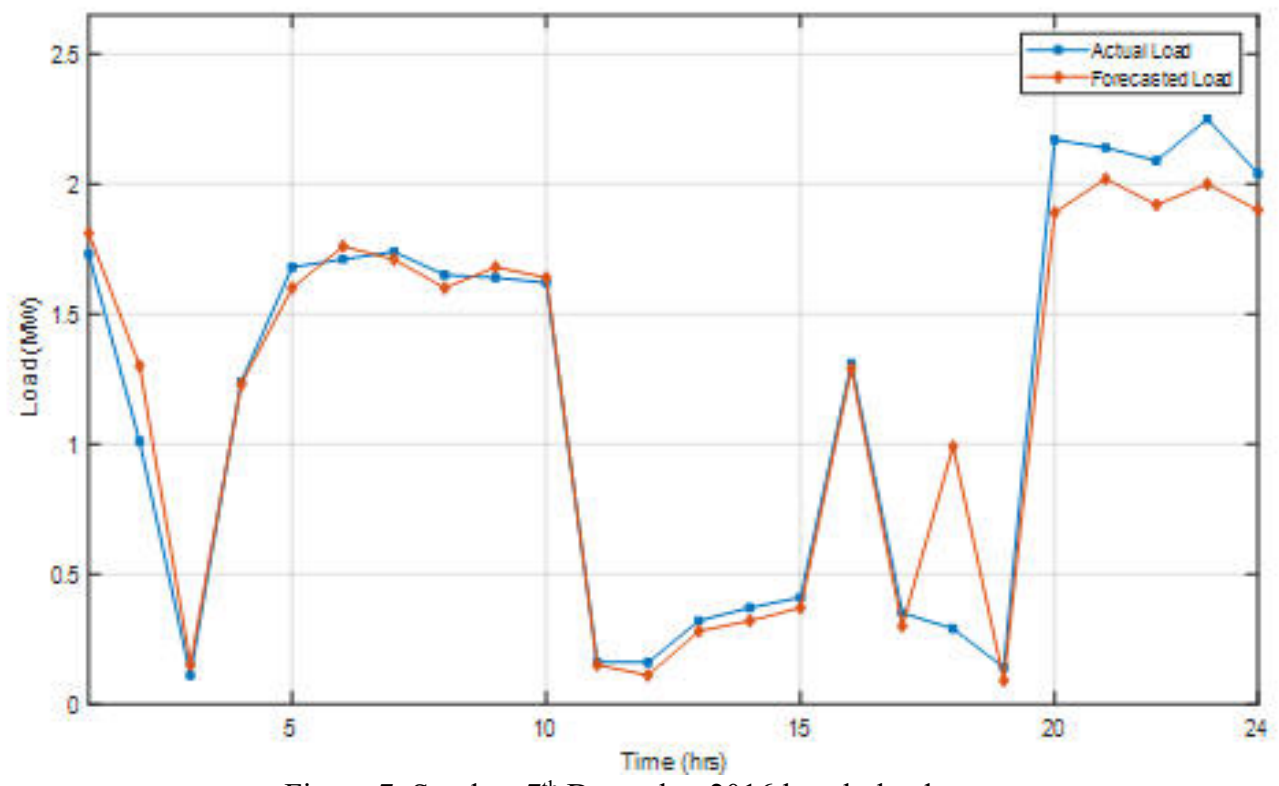

Figure 7: Sunday, $7^{\text {th }}$ December 2016 hourly load curve

Table 4: MAPE and MPE value for the model on the test set

\begin{tabular}{|l|c|c|}
\hline Test Days & MAPE (\%) & MPE (\%) \\
\hline Mon, $1^{\text {st }}$ Dec 2016 & 4.72 & 3.45 \\
\hline Tues, $2^{\text {nd }}$ Dec 2016 & 6.06 & 2.50 \\
\hline Wed, $3^{\text {rd }}$ Dec 2016 & 3.06 & 1.04 \\
\hline Thurs, $4^{\text {th }}$ Dec 2016 & 4.67 & 3.58 \\
\hline Fri, $5^{\text {th }}$ Dec 2016 & 3.00 & 1.50 \\
\hline Sat, $6^{\text {th }}$ Dec 2016 & 3.94 & 2.76 \\
\hline Sun, $7^{\text {th }}$ Dec 2016 & 20.72 & 14.37 \\
\hline Mon, to Sun, Dec 2016 & $\mathbf{6 . 6 0}$ & $\mathbf{4 . 1 7}$ \\
\hline
\end{tabular}

Table 4 presents performance evaluation of the ANN-model developed based on MPE and MAPE. Generally, less value of MAPE and MPE shows a better forecast accuracy, Wednesday shows the least error values of 3.06\% and $1.04 \%$ for MAPE and MPE, respectively. The largest error values were recorded on Sunday with $20.62 \%$ and $14.37 \%$ for MAPE and MPE, respectively. Based on Table 4, it can be seen that the ANN showed higher forecasting error in the days when people have specific start-up activities such as Tuesday and variant activities such as during Sundays. This is probably because of pick-up loads associated with such days. Also, from Table 4, the average MPE and MAPE for this model was found to be $4.17 \%$ and $6.60 \%$ respectively; this implies a high degree of forecasting accuracy for this model in spite of the simplicity of its input variables and the low volume of data used. Notwithstanding the scale sensitivity of MAPE which would often time take extreme values for low volume data, the average MAPE is still small.

\section{Conclusion}

This paper presented application of ANN trained with Bat algorithm to forecast university-based load demand on short term. The forecast results showed that ANN is well suited for this kind of load forecast. Its forecasting reliabilities were evaluated by computing the mean absolute error between the exact and predicted values, the output curves obtained from ANN model developed showed the forecasted load traced out almost same path with the actual consumed in each day of the week. This work when integrated into the power system will go a long way in helping the operator to know the strategies to be adopted once the exact projections of the load expected in future time are known.

\section{References}

Adepoju, G.A., Ogunjuyigbe, S.O.A. and Alawode, K.O. (2007). Application of neural network to load forecasting in Nigerian electrical power system. Pacific Journal of Science and Technology. 8(1):68-72

Christopher A. M and Francis K. K. (2013). Useof artificial neural networks for short term electricity load forecasting of Kenya national grid power system. International Journal of Computer Applications, 63(2): 25- 
30

Jianwei, M., Libin, F, Xuechao, D., \&Yuanying, Q. (2018). Short-term power load forecasting method based on improved exponential smoothing grey model. Mathematical Problems in Engineering, 2018: 1-11

Kalu, C., Ezenugu, I.A and Simeon, O. (2015). Development of Matlab-based software for peak load estimation and forecasting: a case study of faculty of engineering, Imo state university Owerri, Imo state, Nigeria. European Journal of Engineering and Technology, 3(8):20-28

Merkel, G.D., Povinelli,R. J. and Brow, R.H.(2018). Short-term load forecasting of natural gas with deep neural network regression. Energies, 1-20

Olabode, O. E., Okakwu, I. K., Ade-Ikuesan, O. O. and Amuda S. O (2019). A survey on electric load forecasting in Nigerian electrical utility networks. Futo Journal Series, 5(1):127 - 140

Olabode, O. E., Okakwu, I. K., Ade-Ikuesan, O. O., and Fajuke, I. D. (2018). Performance evaluation of mediumterm load forecasting approaches: a case study of Ogun State, Nigeria. Journal of Advances in Science and Engineering,1(2):9-16

Olagoke,M.D., Ayeni,A.A and Hambali, M.A. (2016).Short term electric load forecasting using neural network and genetic algorithm. International Journal of Applied Information Systems, 10(4):22-28

Samuel, I.A., Emmanuel, A., Odigwe, I.A. and Felly-Njoku, F.C. (2017). A comparative study of regression analysis and artificial neural network methods for medium-term load forecasting. Indian Journal of Science and Technology, 10(10):1-7

Sheikh S. K., and Unde M.G. (2012). Short-Term Load Forecasting Using Ann Technique. International Journal of Engineering Sciences\& Emerging Technologies, 1(2): 97-107

Uduak, U., Ini U., Mfon, N., and Emmanuel, B. (2018). Interval type-2 fuzzy neural networks for short-term electric load forecasting: a comparative study. International Journal on Soft Computing, 9(1):1-20 